\title{
n-JORDAN HOMOMORPHISMS
}

\author{
M. ESHAGHI GORDJI
}

(Received 28 November 2008)

\begin{abstract}
Let $n \in \mathbb{N}$ and let $A$ and $B$ be rings. An additive map $h: A \rightarrow B$ is called an $n$-Jordan homomorphism if $h\left(a^{n}\right)=(h(a))^{n}$ for all $a \in A$. Every Jordan homomorphism is an $n$-Jordan homomorphism, for all $n \geq 2$, but the converse is false in general. In this paper we investigate the $n$-Jordan homomorphisms on Banach algebras. Some results related to continuity are given as well.
\end{abstract}

2000 Mathematics subject classification: primary 47B48; secondary 46L05, 46H25. Keywords and phrases: Jordan homomorphism, $n$-homomorphism, Banach algebra.

\section{Introduction and preliminaries}

Let $A, B$ be two rings (algebras). An additive map $h: A \rightarrow B$ is called an $n$-Jordan homomorphism if $h\left(a^{n}\right)=(h(a))^{n}$ for all $a \in A$; it is an $n$-ring homomorphism if $h\left(\prod_{i=1}^{n} a_{i}\right)=\prod_{i=1}^{n} h\left(a_{i}\right)$ for all $a_{1}, a_{2}, \ldots, a_{n} \in A$. If $h: A \rightarrow B$ is a linear $n$-ring homomorphism, we say that $h$ is an $n$-homomorphism. The concept of $n$-homomorphism was studied for complex algebras by Hejazian, Mirzavaziri, and Moslehian [3] (see also [1, 7]). A 2-Jordan homomorphism is a Jordan homomorphism, in the usual sense, between rings. Every Jordan homomorphism is an $n$-Jordan homomorphism, for all $n \geq 2$ (see, for example, [6, Lemma 6.3.2]), but the converse is false, in general. For instance, let $A$ be an algebra over $\mathbb{C}$ and let $h: A \rightarrow A$ be a nonzero Jordan homomorphism on $A$. Then $-h$ is a 3-Jordan homomorphism. It is easy to check that $-h$ is not 2-Jordan homomorphism or 4-Jordan homomorphism. The study of ring homomorphisms between Banach algebras $A$ and $B$ is of interest even if $A=B=\mathbb{C}$. For example the zero mapping, the identity and the complex conjugate are ring homomorphisms on $\mathbb{C}$, which are all continuous. On the other hand the existence of a discontinuous ring homomorphism on $\mathbb{C}$ is well known. More explicitly, if $G$ is the set of all surjective ring homomorphisms on $\mathbb{C}$, then $\operatorname{Card}(G)=2^{\operatorname{Card}(\mathbb{C})}$. In fact, Charnow [2, Theorem 3] proved that there exist $2^{\operatorname{Card}(\mathbb{C})}$ automorphisms for every algebraically closed field $K$. It is also known that if $A$ is a

(C) 2009 Australian Mathematical Society 0004-9727/2009 \$16.00 
uniform algebra on a compact metric space, then there are exactly $2^{\text {Card(C) }}$ complexvalued ring homomorphisms on $A$ whose kernels are nonmaximal prime ideals (see [4, Corollary 2.4]). As an example, take

$$
\mathcal{A}:=\left[\begin{array}{cccc}
0 & \mathbb{R} & \mathbb{R} & \mathbb{R} \\
0 & 0 & \mathbb{R} & \mathbb{R} \\
0 & 0 & 0 & \mathbb{R} \\
0 & 0 & 0 & 0
\end{array}\right] ;
$$

then $\mathcal{A}$ is an algebra equipped with the usual matrix-like operations. It is easy to see that

$$
\mathcal{A}^{3} \neq 0=\mathcal{A}^{4} \text {. }
$$

So any additive map from $\mathcal{A}$ into itself is a 4-Jordan homomorphism, but its kernel does not need to be an ideal of $\mathcal{A}$. Now let $\mathcal{B}$ be the algebra of all $\mathcal{A}$-valued continuous functions from $[0,1]$ into $\mathcal{A}$ with supremum norm. Then $\mathcal{B}$ is an infinite-dimensional Banach algebra, and the product of any four elements of $\mathcal{B}$ is 0 . Since $\mathcal{B}$ is infinitedimensional, there are linear discontinuous maps which are 4-Jordan homomorphisms from $\mathcal{B}$ into itself (see [3]). In this paper we study the continuity of linear $n$-Jordan homomorphisms on $C^{*}$-algebras.

\section{Main result}

By definition, it is obvious that $n$-ring homomorphisms are $n$-Jordan homomorphisms. Conversely, under a certain condition, $n$-Jordan homomorphisms are ring homomorphisms. For example, each Jordan homomorphism $h$ from a commutative Banach algebra $A$ into $\mathbb{C}$ is a ring homomorphism: Fix $a, b \in A$ arbitrarily. Since $h\left((a+b)^{2}\right)=h(a+b)^{2}$ a simple calculation shows that $h(a b+$ $b a)=2 h(a) h(b)$. The commutativity of $A$ implies that $h(a b)=h(a) h(b)$ and hence $h$ is a ring homomorphism. In 1968, Zelazko [8] proved the following theorem (see also [5, Theorem 1.1]).

THEOREM 2.1. Suppose that A is a Banach algebra, which need not be commutative, and suppose that $B$ is a semisimple commutative Banach algebra. Then each Jordan homomorphism $h: \rightarrow B$ is a ring homomorphism.

We prove the following result for 3-Jordan homomorphisms and 4-Jordan homomorphisms on commutative algebras.

Theorem 2.2. Let $n \in\{3,4\}$ be fixed, $A, B$ be two commutative algebras, and let $h: A \rightarrow B$ be an n-Jordan homomorphism. Then $h$ is an $n$-ring homomorphism.

PROOF. First, let $n=3$. Recall that $h$ is additive mapping such that $h\left(a^{3}\right)=(h(a))^{3}$ for all $a \in A$. Replacement of $a$ by $x+y$ results in

$$
h\left(x^{2} y+x y^{2}\right)=h(x)^{2} h(y)+h(x) h(y)^{2} .
$$


Hence, for every $x, y, z \in A$,

$$
\begin{aligned}
h(x y z)= & \frac{1}{2} h\left\{(x+z)^{2} y+(x+z) y^{2}-\left(x^{2} y+x y^{2}+z^{2} y+z y^{2}\right)\right\} \\
= & \frac{1}{2}\left\{h\left[(x+z)^{2} y+(x+z) y^{2}\right]-h\left[x^{2} y+x y^{2}\right]-h\left[z^{2} y+z y^{2}\right]\right\} \\
= & \frac{1}{2}\left\{[h(x+z)]^{2} h(y)+(x+z)[h(y)]^{2}-[h(x)]^{2} h(y)+h(x)[h(y)]^{2}\right. \\
& \left.\quad-[h(z)]^{2} h(y)+h(z)[h(y)]^{2}\right\} \\
= & h(x) h(y) h(z) .
\end{aligned}
$$

This means that $h$ is a 3-ring homomorphism. Now suppose that $n=4$. Then $h$ is additive and $h\left(a^{4}\right)=(h(a))^{4}$ for all $a \in A$. Replace $a$ by $x+y$ in the equality above to get

$$
h\left(4 x^{3} y+6 x^{2} y^{2}+4 x y^{3}\right)=4 h(x)^{3} h(y)+6 h(x)^{2} h(y)^{2}+4 h(x) h(y)^{3} .
$$

Replacing $x$ by $x+z$ in (2.2), we obtain

$$
\begin{aligned}
& h\left\{\left(4 x^{3} y+\right.\right.\left.\left.6 x^{2} y^{2}+4 x y^{3}\right)+\left(4 z^{3} y+6 z^{2} y^{2}+4 z y^{3}\right)+12\left(x^{2} z y+x z^{2} y+x z y^{2}\right)\right\} \\
&=\left(4 h(x)^{3} h(y)+6 h(x)^{2} h(y)^{2}+4 h(x) h(y)^{3}\right)+\left(4 h(z)^{3} h(y)+6 h(z)^{2} h(y)^{2}\right. \\
&\left.\quad+4 h(z) h(y)^{3}\right)+12\left(h(x)^{2} h(z) h(y)+h(x) h(z)^{2} h(y)\right. \\
&\left.\quad+h(x) h(z) h(y)^{2}\right) .
\end{aligned}
$$

Combining (2.2) and (2.3) gives

$$
h\{(x y z)(x+y+z)\}=(h(x) h(y) h(z))(h(x)+h(y)+h(z)) .
$$

Replace $z$ by $-x$ in (2.4) to obtain

$$
h\left(x^{2} y^{2}\right)=h(x)^{2} h(y)^{2}
$$

and replace $y$ by $y+w$ in (2.5) to get

$$
h\left(x^{2} y w\right)=h(x)^{2} h(y) h(w) .
$$

Now replace $x$ by $x+t$ to obtain

$$
h(x t y w)=h(x) h(t) h(y) h(w) .
$$

Hence, $h$ is a 4-ring homomorphism.

By Theorem 2.2 and [1, Theorem 3.2] we deduce the following result.

Corollary 2.3. Let $h: A \rightarrow B$ be a linear involution preserving 3-Jordan homomorphism between commutative $C^{*}$-algebras. Then $h$ is norm contractive (that is, $\|h\| \leq 1)$.

Also, by Theorem 2.2 and [7, Theorem 2.3], we have the following corollary. 
Corollary 2.4. Let $h: A \rightarrow B$ be a linear involution preserving 4-Jordan homomorphism between commutative $C^{*}$-algebras; then $h$ is completely positive. Thus $h$ is bounded.

Now we prove our main theorem.

THEOREM 2.5. Suppose that A is a Banach algebra, which need not be commutative, and suppose that $B$ is a semisimple commutative Banach algebra. Then each 3-Jordan homomorphism $h: A \rightarrow B$ is a 3-ring homomorphism.

PROOF. We prove the theorem in two steps as follows.

Step I. Suppose $B=\mathbb{C}$. We have $h\left(a^{3}\right)=h(a)^{3}$ for all $a \in A$. Replace $a$ by $x+y$ to obtain

$$
h\left(x y x+y x^{2}+y^{2} x+x^{2} y+x y^{2}+y x y\right)=3\left(h(x)^{2} h(y)+h(x) h(y)^{2}\right)
$$

and replace $y$ by $-y$ in (2.7) to get

$$
h\left(-x y x-y x^{2}+y^{2} x-x^{2} y+x y^{2}+y x y\right)=3\left(-h(x)^{2} h(y)+h(x) h(y)^{2}\right) .
$$

By (2.7) and (2.8) we obtain the relation

$$
h\left(x y^{2}+y^{2} x+y x y\right)=3\left(h(x) h(y)^{2}\right) .
$$

Replacing $y$ by $y-z$ in (2.9), we get

$$
\begin{aligned}
& h\left(x y^{2}+x z^{2}-2 x y z+y x y-y x z-z x y+z x z+z^{2} x+y^{2} x-2 y z x\right) \\
& \quad=3\left(h(x)^{2} h(y)+h(x) h(y)^{2}\right)-6 h(x) h(y) h(z) .
\end{aligned}
$$

By (2.9) and (2.10), we obtain

$$
h(y x z+z x y+2 x y z+2 y z x)=6 h(x) h(y) h(z) .
$$

Replacing $z$ by $x$ in (2.11), we get

$$
h\left(3 y x^{2}+x^{2} y+2 x y x=6 h(x)^{2} h(y),\right.
$$

and combining (2.9) and (2.12), we obtain

$$
h\left(x y x+2 y x^{2}\right)=3 h(x)^{2} h(y) .
$$

From (2.8) and (2.13), we conclude that

$$
h\left(y x^{2}-x^{2} y\right)=0 .
$$

Replacing $x$ by $x+z$ in (2.14), we get

$$
h\left(y x^{2}+y z^{2}+2 y x z-x^{2} y-z^{2} y-2 x z y\right)=0,
$$


and from this equality and (2.14) it follows that

$$
h(y x z-x z y)=0 .
$$

Combining (2.11) and (2.15) gives

$$
h(y x z+3 x y z+2 y z x)=6 h(x) h(y) h(z),
$$

and then replacing $z$ by $x$ in (2.16) leads to

$$
h\left(x y x+y x^{2}\right)=2 h(x)^{2} h(y) .
$$

Finally, combining (2.13) and (2.17) to obtain

$$
h\left(y x^{2}\right)=h(y) h(x)^{2}
$$

and then replacing $x$ by $x+z$ in (2.18), we conclude that

$$
h(y x z)=h(y) h(x) h(z)
$$

hence, $h$ is a 3-ring homomorphism.

STEP II. $B$ is arbitrary semisimple and commutative. Let $M_{B}$ be the maximal ideal space of $B$. We associate with each $f \in M_{B}$ a function $h_{f}: A \rightarrow \mathbb{C}$ defined by

$$
h_{f}(a):=f(h(a))
$$

for all $a \in A$. It is easy to see that $h_{f}$ is additive and $h_{f}\left(a^{3}\right)=\left(h_{f}(a)\right)^{3}$ for all $a \in A$. So step I applied to $h_{f}$ implies that $h_{f}$ is a 3-ring homomorphism. By the definition of $h_{f}$, we obtain that

$$
f(h(a b c))=f(h(a)) f(h(b)) f(h(c))=f(h(a) h(b) h(c)) .
$$

Hence

$$
h(a b c)-h(a) h(b) h(c) \in \operatorname{Ker}(f)
$$

for all $a, b, c \in A$ and all $f \in M_{B}$. Since $B$ is assumed to be semisimple, we get $h(a b c)=h(a) h(b) h(c)$ for all $a, b, c \in A$. We thus conclude that $h$ is a 3-ring homomorphism, and the proof is complete.

From now on we consider such $n$-Jordan homomorphisms as are linear.

Corollary 2.6. Suppose that $A, B$ are $C^{*}$-algebras, where $A$ need not be commutative, and suppose that $B$ is semisimple and commutative. Then every involution preserving 3-Jordan homomorphism $h: A \rightarrow B$ is norm contractive (that is, $\|h\| \leq 1)$.

PROOF. It follows from Theorem 2.5 and [1, Theorem 2.1]. 
THEOREM 2.7. Let $h: A \rightarrow B$ be a bounded involution preserving $k$-Jordan homomorphism between $C^{*}$-algebras such that $h\left(a^{*} a\right)=h(a)^{*} h(a)$ for all $a \in A$. Then $h$ is norm contractive (that is, $\|h\| \leq 1$ ).

Proof. From [7, Lemma 2.4],

$$
\begin{aligned}
\|h(a)\|^{4 k+2} & =\left\|\left(h(a)^{*} h(a)\right)^{2 k+1}\right\|=\left\|\left(h(a)^{*} h(a)\right)^{k}\left(h(a)^{*} h(a)\right)\left(h(a)^{*} h(a)\right)^{k}\right\| \\
& =\left\|\left[h(a)\left(h(a)^{*} h(a)\right)^{k}\right]^{*}\left[h(a)\left(h(a)^{*} h(a)\right)^{k}\right]\right\| \\
& =\left\|h(a)\left(h(a)^{*} h(a)\right)^{k}\right\|^{2}=\left\|h(a)\left(h\left(a^{*} a\right)\right)^{k}\right\|^{2} \\
& =\left\|h(a)\left(h\left(a^{*} a\right)^{k}\right)\right\|^{2} \leq\|h(a)\|^{2}\left\|h\left(\left(a^{*} a\right)^{k}\right)\right\|^{2} \\
& \leq\|h\|^{2}\|a\|^{2}\|h\|^{2}\left\|\left(a^{*} a\right)^{k}\right\|^{2} \\
& \leq\|h\|^{4}\|a\|^{4 k+2},
\end{aligned}
$$

for all $a \in A$, which implies that $\|h\| \leq 1$ by taking $(4 k+2)$ th roots.

\section{References}

[1] J. Bracic and M. S. Moslehian, 'On automatic continuity of 3-homomorphisms on Banach algebras', Bull. Malays. Math. Sci. Soc. (2) 30(2) (2007), 195-200.

[2] A. Charnow, 'The automorphisms of an algebraically closed field', Canad. Math. Bull. 13 (1970), 95-97.

[3] Sh. Hejazian, M. Mirzavaziri and M. S. Moslehian, ' $n$-homomorphisms', Bull. Iranian Math. Soc. 31(1) (2005), 13-23.

[4] T. Miura, S.-E. Takahasi and N. Niwa, 'Prime ideals and complex ring homomorphisms on a commutative algebra', Publ. Math. Debrecen 70(3-4) (2007), 453-460.

[5] T. Miura, S.-E. Takahasi and G. Hirasawa, 'Hyers-Ulam-Rassias stability of Jordan homomorphisms on Banach algebras', J. Inequal. Appl. 2005(4) (2005), 435-441.

[6] T. Palmer, 'Banach algebras and the general theory of $*$-algebras', in: Vol. I. Algebras and Banach algebras, Encyclopedia of Mathematics and its Applications, 49 (Cambridge University Press, Cambridge, 1994).

[7] E. Park and J. Trout, 'On the nonexistence of nontrivial involutive $n$-homomorphisms of $C^{*}$ algebras', Trans. Amer. Math. Soc. 361 (2009), 1949-1961.

[8] W. Zelazko, 'A characterization of multiplicative linear functionals in complex Banach algebras', Studia Math. 30 (1968), 83-85.

M. ESHAGHI GORDJI, Department of Mathematics, Semnan University, PO Box 35195-363, Semnan, Iran

e-mail: madjid.eshaghi@gmail.com 